\title{
Estudios genéticos del metabolismo de la warfarina y su utilidad en la toma de decisiones
}

\author{
Genetic studies of warfarin metabolism and its usefulness in decision making
}

\author{
Cristhian F. Ramírez-Ramos ${ }^{1,2 *}$, Gustavo A. Castilla-Agudelo2,3, Jacobo Corrales-Gómez ${ }^{4}$,
} Carlos A. Martínez-Cano y Clara I. Saldarriaga-Giraldo 3,5

${ }^{1}$ Departamento de Cardiología Intervencionista y Hemodinámica, Universidad Pontífica Bolicariana; ${ }^{2}$ Clínica CardioVID; ${ }^{3}$ Departamento de Cardiología Clínica, Universidad Pontifica Bolivariana; ${ }^{4}$ Departamento de Anestesiología, Universidad de Antioquia; ${ }^{5}$ Departamento de Cardiología. Medellín, Colombia

\section{Resumen}

Introducción: Al momento de valorar la necesidad de realizar un reemplazo quirúrgico valvular cardiaco es posible elegir entre una válvula mecánica o una bioprotésica; la elección debe tener presentes los riesgos de la terapia anticoagulante y la necesidad potencial o el riesgo de nuevas intervenciones. La anticoagulación se realiza con antagonistas de la vitamina $K, y$ de estos, la warfarina es el que se prescribe con mayor frecuencia. Por su metabolismo hepático (P450), dicho medicamento tiene múltiples interacciones farmacológicas y no farmacológicas que en ocasiones se convierten en un verdadero problema en la práctica clínica. Hasta la fecha no se recomienda realizar de forma rutinaria una dosificación guiada por genotipo; sin embargo, se requieren estudios genéticos para definir conductas médicas cuando se hace difícil su manejo. Caso clínico: Se describe el caso de una mujer de 37 años portadora de una válvula mitral mecánica por enfermedad reumática, anticoagulada de forma crónica con warfarina; sin embargo, durante el seguimiento tuvo múltiples consultas a urgencias (entre dos y tres veces por mes) por niveles de anticoagulación en rangos subterapéuticos, como valores elevados de INR. Presentó infarto agudo de miocardio con coronarias sanas e isquemia cerebral transitoria en contexto de INR bajo, considerados así de etiología tromboembólica. Por estas dificultades se decidió realizar la medición de los niveles de factor II de la coagulación, el cual fue normal a pesar del uso del medicamento, por lo que se sospechó resistencia al fármaco. Se solicitó estudio genético que mostró genotipo asociado con actividad enzimática reducida o normal de la CYP2C9, además un genotipo WARF CYP2C9 *1/*2 y WARF VKORC1 A/A, con lo cual se concluyó que la paciente presentaba un comportamiento metabólico divergente para warfarina. Se decidió realizar un reemplazo de válvula mecánica por válvula bioprotésica, con el objetivo de suspender el uso de la warfarina. La paciente presentó una evolución clínica satisfactoria. Conclusiones: La farmacogenética ha logrado identificar polimorfismos en los genes implicados en el metabolismo de la warfarina, los cuales están relacionados con riesgo de sangrado. Estas variantes se encuentran relacionadas con los genes CYP2C9, CYP4F2 y VKORC1. Si bien no se ha demostrado un impacto clínico en los ajustes de warfarina guiados por genotipo, dichos exámenes se hacen necesarios en algunos casos para sugerir un cambio en la dosis del medicamento o su suspensión definitiva.

Palabras clave: Polimorfismo genético. Warfarina. Anticoagulantes. Válvula mitral

\section{Correspondencia:}

*Cristhian F. Ramírez-Ramos

E-mail: cristhianramos1989@ hotmail.com
Disponible en internet: 22-02-2022 Rev Colomb Cardiol. 2022;29(1):100-105
Rev Colomb Cardiol. 2022;29(1):100-105
www.rccardiologia.com 0120-5633 / @ 2020 Sociedad Colombiana de Cardiología y Cirugía Cardiovascular. Publicado por Permanyer. Este es un artículo open access bajo la licencia CC BY-NC-ND (http://creativecommons.org/licenses/by-nc-nd/4.0/).

Fecha de recepción: 11-01-2020

Fecha de aceptación: 11-09-2020

DOI: 10.24875/RCCAR.M22000124 


\section{Abstract}

Introduction: When assessing the need for a cardiac valvular surgical replacement, it is possible to choose between a mechanical or bioprosthetic valve; The choice must take into account the risks of anticoagulant therapy and the potential need and/or risk of new interventions. Anticoagulation is performed with vitamin $\mathrm{K}$ antagonists and warfarin is the most commonly prescribed of these. This medicine due to its hepatic metabolism (P450) has multiple pharmacological and non-pharmacological interactions that sometimes become a real problem in clinical practice. To date, it is not recommended to routinely perform a genotype-guided dosage. However, such genetic studies are necessary to define medical behaviors when handling is difficult. Case report: It is described a case of a 37-year-old woman with a mechanical mitral valve due to rheumatic disease, chronically anticoagulated with warfarin; however, during the follow-up with multiple emergency consultations (2-3 times per month) for anticoagulation levels in subtherapeutic ranges such as elevated INR levels. She presented acute myocardial infarction with healthy coronary arteries and transient cerebral ischemia in the context of low INR thus considered thromboembolic etiology. Due to these difficulties, it was decided to measure coagulation factor II levels, which was normal despite the use of the drug, suspecting drug resistance. A genetic study was requested that showed genotype associated with reduced or normal CYP2C9 enzymatic activity, plus a WARF CYP2C9 * $1{ }^{*} 2$ and WARF VKORC1 A/A genotype concluding that the patient presented a divergent metabolic behavior for warfarin. It was decided to perform a mechanical valve replacement with a bioprosthetic valve, in order to suspend the use of warfarin. The patient presented a satisfactory clinical evolution. Conclusions: Pharmacogenetics has managed to identify polymorphisms in the genes involved in warfarin metabolism, which are related to bleeding risk. These variants are related to the CYP2C9, CYP4F2 and VKORC1 genes. Although no clinical impact has been demonstrated in genotype-guided warfarin adjustments, such tests are necessary in some cases to suggest a change in the dose of the medication or the definitive suspension.

Keywords: Genetic polymorphism. Warfarin. Anticoagulants. Mitral valve

\section{Introducción}

Al momento de valorar la necesidad de realizar un reemplazo quirúrgico valvular cardiaco es posible elegir entre una válvula mecánica o una válvula bioprotésica. La elección del tipo de válvula cardiaca protésica para el reemplazo quirúrgico es un proceso en el cual es necesario tener presentes los riesgos de la terapia anticoagulante y la necesidad potencial o el riesgo de nuevas intervenciones, además de las preferencias del paciente. El equipo médico debe contemplar la comorbilidad del paciente, la adherencia a la terapia farmacológica, la edad y la esperanza de vida ${ }^{1,2}$.

Teniendo en cuenta el proceso de deterioro de las prótesis biológicas y la necesidad de reintervención a mediano plazo, en múltiples situaciones (principalmente en pacientes jóvenes) se decide emplear las prótesis mecánicas; sin embargo, para hacer uso de esta alternativa terapéutica se requiere anticoagulación indefinida para evitar la trombosis de la prótesis y eventos tromboembólicos ${ }^{3}$. Las pruebas no han mostrado buenos resultados con anticoagulantes directos orales ${ }^{4}$, y por ello solo está indicado el uso de antagonistas de la vitamina $K^{1}$. En este grupo farmacológico, la warfarina es el fármaco más utilizado; sin embargo, presenta múltiples interacciones farmacológicas y no farmacológicas, y tiene un estrecho rango terapéutico y una marcada variabilidad interindividual, lo que limita su uso, y se requiere un control riguroso en su administración a través de la medición del INR (International Normalized Ratio) para evitar accidentes hemorrágicos por sobreanticoagulación o trombóticos por subterapéutica del medicamento ${ }^{5}$. Uno de los aspectos que influencian la respuesta a la warfarina es el genético, con polimorfismos conocidos por alterar su rango terapéutico en una manera dependiente de la dosis ${ }^{6}$. Si bien hasta la fecha no se recomienda realizar de forma rutinaria una dosificación guiada por genotipo para la administración de un antagonista de la vitamina $\mathrm{K}^{7}$, hay algunos datos que muestran grupos poblacionales (afroamericanos) con mayor riesgo derivado de la presencia más común de dichas alteraciones genéticas, lo cual sugiere la necesidad de algoritmos de manejo en estos pacientes ${ }^{8}$. Por otro lado, estas pruebas farmacológicas pueden ayudar a tomar conductas terapéuticas en pacientes con uso crónico del medicamento, pero que tienen dificultad en el control.

Se presenta un caso en el cual una mujer portadora de una válvula mitral mecánica requirió un cambio por una válvula bioprotésica en contexto de importante labilidad del INR guiado por pruebas genéticas que permitieron establecer un polimorfismo en el metabolismo de la warfarina, que ocasionaba un comportamiento metabólico divergente para este medicamento. El objetivo es presentar la utilidad de las pruebas 
genéticas del metabolismo de la warfarina y la dosificación guiada por estas en la práctica clínica.

\section{Caso clínico}

Mujer de 37 años, con antecedentes de fiebre reumática, diagnosticada en la adolescencia, y colitis ulcerativa en tratamiento inmunosupresor. A los 31 años, durante su primer embarazo, presentó edema pulmonar espontáneo posterior a la cirugía al ser sometida a cesárea urgente (a las 31 semanas de gestación) en contexto de oligoamnios. Sus estudios mostraron insuficiencia mitral excéntrica grave tipo IIIA con dilatación del ventrículo izquierdo y signos indirectos de hipertensión pulmonar leve. Por persistencia de los síntomas, se realizó reemplazo valvular mitral por prótesis mecánica número 29, mediante cirugía cardiaca mínimamente invasiva, sin complicaciones. Sin embargo, desde entonces requirió múltiples consultas y controles médicos en clínica de anticoagulación (2-3 por semana) por ser difícil mantener su nivel de INR en rango terapéutico objetivo, llamando la atención que con pequeños ajustes tenía respuestas desproporcionadas (con dosis semanales promedio entre 57.5 y $65 \mathrm{mg}$ de warfarina). Durante ese mismo año presentó isquemia cerebral transitoria y 5 años después un infarto agudo de miocardio sin elevación del segmento ST; en la coronariografía no se identificó enfermedad coronaria aterosclerótica. Ambos cuadros ocurrieron con INR subterapéuticos, probablemente explicados por microembolia.

Las consultas se volvieron cada vez más frecuentes, razón por lo cual se decidió realizar la medición de los niveles de factor II de la coagulación, con unos valores reportados en rango de la normalidad a pesar del uso de la warfarina, por lo que se sospechó resistencia a los antagonistas de la vitamina K. Se solicitó estudio farmacogenético, que mostró un genotipo asociado con actividad enzimática reducida o normal de la CYP2C9, y además un genotipo WARF CYP2C 9 *1/*2 y WARF VKORC1 A/A, lo que sugirió que podría requerirse una dosis moderada a baja de warfarina para mantener un nivel óptimo de INR. Sin embargo, el alelo CYP4F2*3: *1/*3 (rs2108622T) también se encontró presente; se asoció a necesidades de incremento de dosis de warfarina para lograr efectividad clínica. Por todo lo anterior, se concluyó que la paciente presentaba un comportamiento metabólico divergente para los antagonistas de la vitamina $\mathrm{K}$, dado por los polimorfismos (por un lado, aumentan la sensibilidad, pero por otro lado inducen resistencia) y reforzado por su comportamiento clínico.

Con base en ello, y considerando la gran variabilidad del INR de la paciente, los episodios trombóticos sistémicos, el alto riesgo de trombosis de la prótesis mecánica, los resultados del estudio genético que predecían dificultades en continuar con el uso de antagonistas de la vitamina $\mathrm{K}$ y la imposibilidad de usar otra estrategia de anticoagulación, como heparinas por vía subcutánea (a largo plazo pueden favorecer la fibrosis en el tejido) y demás anticoagulantes orales directos, se decidió realizar un cambio de válvula mecánica por válvula biológica, procedimiento que transcurrió sin complicaciones y que tuvo una evolución clínica que ha sido satisfactoria a la fecha.

\section{Discusión}

Se presenta un caso clínico en el que el estudio farmacogenético en una paciente con dificultad para lograr niveles óptimos de anticoagulación permitió guiar la conducta terapéutica.

En muchas oportunidades es un gran desafío seleccionar el tipo de prótesis cardiaca adecuada para un paciente, y la literatura es limitada respecto a estudios que comparan resultados con tipos específicos de válvulas mecánicas o bioprotésicas ${ }^{9}$. El sangrado es un problema común en los pacientes con válvulas cardiacas protésicas, complicación que se presenta con mayor frecuencia en pacientes con prótesis mecánicas debido a la necesidad de anticoagulación oral de forma indefinida ${ }^{10}$, por el hecho de que todos estos pacientes tienen un riesgo latente de presentar un evento trombótico; aunque los datos sean limitados, hay información que sugiere que este riesgo es menor con las válvulas biológicas ${ }^{1}$. A pesar de la mejoría en el diseño de las válvulas, la incidencia de trombosis de la válvula mecánica se reporta entre el $0.5 \%$ y el $8 \%{ }^{11}$. Hasta la fecha solo se tiene disponible la warfarina como anticoagulante oral en este tipo de pacientes, medicamento que ha sido usado por más de 50 años para dicho fin.

Utilizar un antagonista de la vitamina K, como la warfarina, es un reto clínico constante por su margen terapéutico estrecho; además, la dosificación se ve afectada por factores clínicos, dieta, interacciones medicamentosas y polimorfismos genéticos; estos últimos son sumamente cruciales, pues estos pacientes pueden tener una mayor sensibilidad al medicamento, como otros asociados a resistencia ${ }^{6}$. La farmacogenética ha logrado identificar polimorfismos en los genes 
implicados en el metabolismo de la warfarina, los cuales están relacionados con riesgo de sangrado. Estas variantes se encuentran asociadas con los genes CYP2C9, CYP4F2 y VKORC1 12-14. En población antioqueña se ha documentado que la variación en la respuesta es explicada por las variantes VKORC1-1639G/A en un $11.3 \%$ y por CYP2C9*1, *2 y *3 en un $7.1 \%{ }^{15}$. En Colombia se han validado algoritmos farmacogenéticos que permiten un ajuste de dosis más rápido y con menor variabilidad ${ }^{16}$. Sin embargo, la evidencia del beneficio clínico de esta aproximación ha sido conflictiva, pues otros estudios no han mostrado beneficio en ajustar la dosis por el método farmacogenético, comparado con la estrategia estándar, y por esto no se recomienda realizarlo de forma rutinaria ${ }^{17,18}$. Por otro lado, se ha encontrando que el polimorfismo CYP4F2 1347 G>A aumenta cinco veces más el riesgo de trombosis valvular ${ }^{19}$, lo que evidencia que estas alteraciones no solo predicen la respuesta terapéutica a la anticoagulación. Este es un campo de continua investigación y es posible que, si esta misma asociación se encuentra en otras poblaciones, el perfil genético del paciente pueda convertirse en uno de los factores de peso antes de definir el tipo de válvula a implantar para evitar complicaciones del manejo farmacológico, como reintervenciones que por lo general son altamente mórbidas. En la tabla 1 se resumen los estudios de algoritmos basados en farmacogenética.

En este reporte se presenta el caso de una paciente que contaba con un metabolismo divergente para los antagonistas de la vitamina $\mathrm{K}$ con polimorfismos que, por un lado, aumentaban la sensibilidad, pero por otro inducían resistencia. Isaza, et al. ${ }^{25}$ cuentan con una serie casos de 10 pacientes con sensibilidad aumentada a la warfarina (dosis $\leq 15 \mathrm{mg} / \mathrm{semana}$ ), de los cuales encontraron que el $100 \%$ tenían entre uno y tres marcadores genéticos de susceptibilidad. Cifuentes, et al. ${ }^{26}$ recopilaron los datos demográficos y clínicos de 130 pacientes colombianos y buscaron determinar, a partir de estudios genéticos, la exactitud del análisis farmacogenético de los principales polimorfismos implicados en el metabolismo de la warfarina; concluyen que los polimorfismos en los genes CYP2C9 y VKORC1 permitieron explicar un $52.8 \%$ de la variación de la dosis de la warfarina, es decir, que a mayor edad o mayor número de mutaciones, mayor es la probabilidad de encontrar sensibilidad aumentada a la warfarina. Un estudio similar realizado por Palacio, et al. ${ }^{15}$, en Antioquia, Colombia, con 191 pacientes, indicó que un $38.2 \%$ de la varianza de la dosis de warfarina fue explicada por un modelo dependiente de la edad y los polimorfismos ya mencionados.

El caso que se presenta es de particular interés por el hecho de encontrar en la paciente alteraciones múltiples de resistencia y aumento de la respuesta al medicamento con la expresión fenotípica final de tener una marcada variación con pequeñas dosis del fármaco, y con la imposibilidad de lograr el objetivo terapéutico. Este tipo de alteraciones, en especial la resistencia a la warfarina, pueden ser de dos tipos: adquiridas (habitualmente debido a alimentos con alto contenido de vitamina $\mathrm{K}$, o alteraciones en el metabolismo del anticoagulante, como disminución de la absorción o aumento del aclaramiento) o hereditarias (extremadamente raras, asociadas a la presencia de una enzima anormal o un receptor que muestra una afinidad disminuida por los cumarínicos o aumentada por la vitamina $\mathrm{K})^{27}$. Isaza, et al. ${ }^{25}$ también reportan tres casos de resistencia (dosis $\geq 70 \mathrm{mg} / \mathrm{semana}$ ) al fármaco, de los cuales dos estaban en contexto de uso concomitante de anticonvulsivantes (potentes inductores de la enzima CYP2C9), pero el tercero presentaba una mutación del gen VKORC1 y, sin embargo, se comportaba como resistente al anticoagulante (similar al presente caso). Los autores de dicho artículo consideraron que estuviese asociado con un trastorno trombofílico de base.

Sospechamos que la mayor sensibilidad y la resistencia a la warfarina en esta paciente se deban a polimorfismos genéticos; hasta ahora no se ha demostrado otro factor diferente a lo ya mencionado que pueda alterar el metabolismo de dicho medicamento. Lo cierto es que, ante el riesgo persistente de sangrado y trombosis en vista de la labilidad del INR, la alternativa terapéutica más segura para la paciente fue el reemplazo de la prótesis valvular mecánica por una biológica.

Con las pruebas clínicas y la evidencia reciente, para nadie es un secreto la disminución del uso de la warfarina. Por esto, el escenario donde sigue siendo la única alternativa es en el del cambio valvular mecánico. En estos pacientes debería realizarse la genotipificación para variantes de CYP2C9 y VKORC1 teniendo en cuenta el trabajo de Gálvez, et al. ${ }^{16}$, como los estudios genéticos realizados respecto a polimorfismos de la warfarina en nuestra población. Planteamos, en concordancia con el estudio de Gálvez et al. ${ }^{16}$, el siguiente algoritmo de dosificación: raíz cuadrada de la dosis de warfarina $(\mathrm{mg})=9.672-(0.02$ * edad) $-(0.404$ * sexo $)-(0.794$ * VKORC1) $-(0.607$ * CYP2C9); con la edad definida en años y el sexo 
Tabla 1. Estudios con utilización de algoritmos farmacogenéticos de dosificación de warfarina

\begin{tabular}{|c|c|c|c|}
\hline $\begin{array}{l}\text { Autor y año de } \\
\text { publicación }\end{array}$ & Lugar & Tipo de estudio (n) & Resultados \\
\hline Pirmohamed et al., $2013^{20}$ & $\begin{array}{l}\text { Suecia y Reino } \\
\text { Unido }\end{array}$ & $\begin{array}{l}\text { Aleatorizado, controlado, } \\
\text { pragmático, un cegamiento (455) }\end{array}$ & $\begin{array}{l}\text { Tiempo de rango terapéutico } 67 \% \text { en el grupo } \\
\text { guiado por genotipo vs. } 60 \% \text { (IC95\%: } 3.3-10 ; \mathrm{p}< \\
0.001 \text { ); menos incidencia de anticoagulación } \\
\text { excesiva. El tiempo medio para alcanzar el INR } \\
\text { fue de } 21 \text { días para el grupo guiado por genotipo } \\
\text { vs. } 29 \text { días. }\end{array}$ \\
\hline Gage et al., $2017^{21}$ & Estados Unidos & Aleatorizado, multicéntrico (1650) & $\begin{array}{l}\text { Resultado primario sangrado mayor, INR } 4 \text { o más, } \\
\text { y muerte a } 30 \text { días, enfermedad tromboembólica } \\
\text { con prueba objetiva en } 60 \text { días: } 10.8 \% \text { en el } \\
\text { grupo guiado por fenotipo vs. } 14.7 \% \text { en el grupo } \\
\text { de manejo médico (IC95\%: } 0.56-0.95 \text { ) }\end{array}$ \\
\hline Gálvez et al., $2018^{16}$ & Bogotá & $\begin{array}{l}\text { Cohorte de generación (152) y de } \\
\text { validación (87) }\end{array}$ & $\begin{array}{l}\text { Dosis semanal menos en pacientes VKORC1-1639 } \\
\text { AA y metabolizadores pobres CYP2C } 9^{*} 2 /{ }^{*} 2,{ }^{*} 2 /{ }^{*} 3 \text {. } \\
\text { Dosis } 2.3 \text { mayores para pacientes con genotipo } \\
\text { salvaje VKORC1/CYP2C9 }\end{array}$ \\
\hline Xu et al., $2018^{22}$ & Asia & Aleatorizado, doble ciego (201) & $\begin{array}{l}\text { Tasa libre de eventos tromboembólicos y } \\
\text { sangrado mayor fue } 97 \% \text { en el grupo guiado por } \\
\text { fenotipo. Tiempo para alcanzar dosis estable de } \\
33 \text { vs. } 42 \text { días, con tiempo de rango terapéutico } \\
\text { de } 47.2 \text { vs. } 47.4 \%\end{array}$ \\
\hline Tse et al., $2018^{23}$ & No limitado & $\begin{array}{l}\text { Revisión sistemática y } \\
\text { metaanálisis (2626) }\end{array}$ & $\begin{array}{l}\text { Tiempo para lograr INR terapéutico estable } 5.9 \\
\text { días menos vs. terapia convencional, } \\
\text { disminución relativa de INR }>4 \text { de } 13 \% \text {, } \\
\text { sangrado } 18 \% \text {, sin diferencias en muerte o } \\
\text { tromboembolia }\end{array}$ \\
\hline Yang et al., $2019^{24}$ & No limitado & Metaanálisis (4.852) & $\begin{array}{l}\text { Dosis guiada por fenotipo se asoció con mayor } \\
\text { porcentaje de tiempo en rango terapéutico, más } \\
\text { pacientes en dosis estables }>1 \text { mes de } \\
\text { seguimiento, tiempo más corto de INR terapéutico } \\
\text { y disminución del riesgo de sangrado mayor }\end{array}$ \\
\hline
\end{tabular}

IC95\%: intervalo de confianza del 95\%; INR: International Normalized Ratio.

codificado 1 para hombres y 2 para mujeres; VKORC1 codificado 1 para GG, 2 para $G A$ y 3 para $A A$; y CYP2C9 codificado 1 para *1/*1, 2 para ${ }^{*} 1 /{ }^{*} 2,{ }^{*} 1 /{ }^{*} 30$ ${ }^{\star} 2 /{ }^{*} 2$, y 3 para $2^{*} / 3^{*}$.

\section{Conclusiones}

Ante un paciente que requiere obligatoriamente anticoagulación oral crónica con warfarina y no se logre obtener valores estables de INR es necesario considerar que la dieta, la asociación con otros fármacos y la genética del paciente son los factores que primordialmente intervienen en la resistencia y la sensibilidad a este fármaco. Es posible considerar la realización de pruebas genéticas con el objetivo de tomar conductas, aunque no es correcto recurrir a dichos exámenes de forma sistemática.

\section{Financiamiento}

El trabajo no fue soportado por ninguna beca ni por fondos de la industria farmacéutica.

\section{Conflicto de intereses}

Los autores declaran la ausencia de conflictos de intereses con respecto al reporte del presente caso.

\section{Responsabilidades éticas}

Protección de personas y animales. Los autores declaran que para esta investigación no se han realizado experimentos en seres humanos ni en animales. 
Confidencialidad de los datos. Los autores declaran que han seguido los protocolos de su centro de trabajo sobre la publicación de datos de pacientes.

Derecho a la privacidad y consentimiento informado. Los autores han obtenido el consentimiento informado del paciente $y / 0$ sujeto referidos en el artículo.

\section{Bibliografía}

1. Nishimura RA, Otto CM, Bonow RO, Carabello BA, Erwin JP Guyton RA, et al. 2014 AHA/ACC Guideline for the management of patients with valvular heart disease: a report of the American College of Cardiology/American Heart Association Task Force on Practice Guidelines. J Am Coll Cardiol. 2014;63:e57-185.

2. Baumgartner H, Falk V, Bax JJ, De Bonis M, Hamm C, Holm PJ, et al. 2017 ESC/EACTS Guidelines for the management of valvular heart disease. Eur Heart J. 2017;21:2739-91.

3. Nishimura RA, Otto CM, Bonow RO, Carabello BA, Erwin JP Fleisher LA, et al. 2017 AHA/ACC Focused Update of the 2014 AHA ACC Guideline for the management of patients with valvular heart disease: a report of the American College of Cardiology/American Heart Association Task Force on Clinical Practice Guidelines. J Am Coll Cardiol. 2017;70:252-89

4. Eikelboom JW, Connolly SJ, Brueckmann M, Granger CB, Kappetein AP Mack MJ, et al. Dabigatran versus warfarin in patients with mechanical heart valves. N Engl J Med. 2013;369:1206-14.

5. Jacobs LG. Warfarin pharmacology, clinical management, and evaluation of hemorrhagic risk for the elderly. Cardiol Clin. 2008;26:157-67.

6. Weeke PE. Pharmacogenetics in cardiovascular medicine. Adv Pharmacol San Diego Calif. 2018;83:333-60.

7. Belley-Cote EP, Hanif H, D'Aragon F, Eikelboom JW, Anderson JL, Borgman $\mathrm{M}$, et al. Genotype-guided versus standard vitamin $\mathrm{K}$ antagonist dosing algorithms in patients initiating anticoagulation. A systematic review and meta-analysis. Thromb Haemost. 2015;114:768-77.

8. Shendre A, Dillon C, Limdi NA. Pharmacogenetics of warfarin dosing in patients of African and European ancestry. Pharmacogenomics. 2018;19:1357-71.

9. Gott VL, Alejo DE, Cameron DE. Mechanical heart valves: 50 years of evolution. Ann Thorac Surg. 2003;76:S2230-9.

10. Hammermeister K, Sethi GK, Henderson WG, Grover FL, Oprian C Rahimtoola SH. Outcomes 15 years after valve replacement with a mechanical versus a bioprosthetic valve: final report of the Veterans Affairs randomized trial. J Am Coll Cardiol. 2000;36:1152-8.

11. Gürsoy MO, Kalçık M, Yesin M, Karakoyun S, Bayam E, Gündüz S, et al. A global perspective on mechanical prosthetic heart valve thrombosis: diagnostic and therapeutic challenges. Anatol J Cardiol. 2016:16:980-9.

12. Shikata E, leiri I, Ishiguro S, Aono H, Inoue K, Koide T, et al. Association of pharmacokinetic (CYP2C9) and pharmacodynamic (factors II, VII, IX and $\mathrm{X}$; proteins $\mathrm{S}$ and $\mathrm{C}$; and gamma-glutamyl carboxylase) gene variants with warfarin sensitivity. Blood. 2004;103:2630-5.

13. Schwarz UI, Ritchie MD, Bradford Y, Li C, Dudek SM, Frye-Anderson A et al. Genetic determinants of response to warfarin during initial anticoagulation. N Engl J Med. 2008;358:999-1008.

14. Al-Eitan LN, Almasri AY, Khasawneh RH. Effects of CYP2C9 and VKORC1 polymorphisms on warfarin sensitivity and responsiveness during the stabilization phase of therapy. Saudi Pharm J. 2019;27:484-90.

15. Palacio L, Falla D, Tobon I, Mejia F, Lewis JE, Martínez AF, et al. Pharmacogenetic impact of VKORC1 and CYP2C9 allelic variants on warfarin dose requirements in a Hispanic population isolate. Clin Appl Thromb. 2010;16:83-90.

16. Gálvez JM, Restrepo CM, Contreras NC, Alvarado C, Calderón-Ospina C-A, Peña N, et al. Creating and validating a warfarin pharmacogenetic dosing algorithm for Colombian patients. Pharmacogenomics Pers Med. 2018;11:169-78.

17. Belley-Cote EP, Hanif H, D'Aragon F, Eikelboom JW, Anderson JL, Borgman M, et al. Genotype-guided versus standard vitamin K antagonist dosing algorithms in patients initiating anticoagulation. A systematic review and meta-analysis. Thromb Haemost. 2015;114:768-77.

18. Stergiopoulos K, Brown DL. Genotype-guided vs. clinical dosing of warfarin and its analogues: meta-analysis of randomized clinical trials. JAMA Intern Med. 2014;174:1330-8.

19. Sr K, Bharath G, Jain S, Moorthy N, Manjunath SC, Christopher R. Prosthetic valve thrombosis - association of genetic polymorphisms of VKORC1, CYP2C9 and CYP4F2 genes. Medicine (Baltimore). 2019;98:e14365

20. Pirmohamed $\mathrm{M}$, Burnside $\mathrm{G}$, Eriksson $\mathrm{N}$, Jorgensen $\mathrm{AL}$, Toh $\mathrm{CH}$ Nicholson $\mathrm{T}$, et al. A randomized trial of genotype-guided dosing of warfarin. N Engl J Med. 2013;369:2294-303.

21. Gage BF, Bass AR, Lin H, Woller SC, Stevens SM, Al-Hammadi N, et al. Effect of genotype-guided warfarin dosing on clinical events and anticoagulation control among patients undergoing hip or knee arthroplasty. JAMA. 2017;318:1115-24.

22. Xu Z, Zhang S-Y, Huang M, Hu R, Li J-L, Cen H-J, et al. Genotype-guided warfarin dosing in patients with mechanical valves: a randomized controlled trial. Ann Thorac Surg. 2018;106:1774-81.

23. Tse G, Gong M, Li G, Wong SH, Wu WKK, Wong WT, et al. Genotype-guided warfarin dosing vs. conventional dosing strategies: a systematic review and meta-analysis of randomized controlled trials. $\mathrm{Br} \mathrm{J}$ Clin Pharmacol. 2018:84:1868-82.

24. Yang T, Zhou Y, Chen C, Lu M, Ma L, Cui Y. Genotype-guided dosing versus conventional dosing of warfarin: a meta-analysis of 15 randomized controlled trials. J Clin Pharm Ther. 2019;44:197-208.

25. Isaza C, Henao J, Beltrán L. Resistance and sensibility to warfarine. Investig Andina. 2010;12:30-40.

26. Cifuentes RA, Murillo-Rojas J, Avella-Vargas E. Prediction of sensitivity to warfarin based on VKORC1 and CYP2C9 polymorphisms in patients from different places in Colombia. Biomed Rev Inst Nac Salud. 2016;36:91-100.

27. Castelhanos Rojas $R$, Ferrer Herrera IM, Segura Pujal LA Ojeda Matías MR, Alfonso Hernández MC. Resistencia y sensibilidad a la warfarina. Rev Arch Med Camagüey. 2014;18:226-39. 